\title{
Post-action determinants of the reported time of conscious intentions
}

\author{
Davide Rigoni $^{1}$, Marcel Brass ${ }^{2}$ and Giuseppe Sartori ${ }^{3}$ \\ 1 Department of Developmental Psychology and Socialization, Università degli Studi di Padova, Padova, Italy \\ 2 Department of Experimental Psychology, Ghent University, Ghent, Belgium \\ ${ }^{3}$ Department of General Psychology, Università degli Studi di Padova, Padova, Italy
}

\section{Edited by:}

Francisco Barceló,

University of Illes Balears, Spain

\section{Reviewed by:}

Ulrike Krämer,

University of Magdeburg, Germany

Nicholas Yeung,

University of Oxford, UK

*Correspondence:

Davide Rigoni,

Department of Socialization and

Developmental Psychology, Università

degli Studi di Padova, via Venezia 8,

35131 Padova, Italy.

e-mail: davide.rigoni@unipd.it
The question of whether our behavior is guided by our conscious intentions is gaining momentum within the field of cognitive neuroscience. It has been demonstrated that the subjective experience that conscious intentions are the driving force of our actions, is built partially on a post hoc reconstruction. Our hypothesis was that this reconstructive process is mediated by an action-monitoring system that compares the predicted and the actual sensory consequences of an action. We applied event-related potentials (ERP) to a variant of the Libet's task in which participants were asked to press a button and to report the time of decision-will judgment (W) - to press. We provided delayed auditory feedbacks after participants' action to signify an action time later than the actual action. We found that auditory feedbacks evoked a negative component in the 250-300 time range, namely action-effect negativity $\left(N_{\mathrm{AE}}\right)$, that is thought to reflect the activity of a system that detects violation from expectancies. We showed that the amplitude of the $N_{A E}$ was sensitive to the delay of the auditory feedback, with a larger amplitude for more delayed feedbacks. Furthermore, changes in the $N_{\mathrm{AE}}$ were also associated with changes in the reportedW. These results not only confirm that we infer the time we decided to act from events occurring after the response, but these results also indicate that the subjective experience of when an action is decided is influenced by the activity of an action-monitoring system that detects mismatches between predicted and actual sensory consequences of the actions.

Keywords: intentions, conscious decisions, action-monitoring, action-effect negativity, free will

\section{INTRODUCTION}

In our daily lives, we have the impression that we are able to choose freely and consciously among different possible courses of actions. Constantly we have the impression that our behavior is guided, at least in many circumstances, by our conscious decisions, which are a sort of 'driving force' of our behavior. In large part, that's presumably because we feel that our conscious decision to act precedes the action itself. For example, when we decide to turn on the TV, we feel clearly that the action of actually turning on the TV is preceded (and caused) by our conscious intention to turn on the TV. Given that the cause always precedes the effect, we feel that our conscious decision caused the TV to be turned on. However, recent findings in psychology and neuroscience have shown that the relationship between conscious intentions behavior is rather more complex.

The question of whether our actions are caused by conscious intentions is intertwined with the question of free will, which has been traditionally conducted only in philosophical and speculative terms. In the last decades, however, the problem of free will has become a neurophysiological one and a great number of studies have attempted a scientific investigation of cognitive and neural mechanisms underlying free will. In order to avoid any confusion about what we intend here by the term 'free will', it must be said that when neuroscientists argue about free will, they actually mean the 'feeling of having a free will', or the 'perception of volition'
(Hallett, 2007). None of the studies conducted so far provide tools for answering the question whether or not free will (in philosophical terms) exists: this is still unproven and perhaps unprovable (Baumeister, 2008). Rather, they illuminate the relationship between brain mechanisms involved in our subjective experience of what appear as conscious and voluntary decisions or intentions to act (Libet et al., 1983; Haggard and Eimer, 1999; Haynes et al., 2007; Soon et al., 2008).

The first attempt of a scientific investigation into the sense of volition has been done by Libet et al. (1983), who conducted pioneering studies on consciousness and intentional acts. In a typical Libet's experiment, subjects sat in front of a clock with a rapidly moving spot and they were asked to move their index finger or their wrist at will. Subsequently, they were asked to report what time it was (i.e. where the spot was) when they had the first subjective experience of intending to act. This reported time is called the will judgment (W). At the same time, Libet et al. (1983) recorded the electrical brain activity and movement-related cortical potentials were assessed to determine the timing of activity of the brain. The critical finding was that individuals' voluntary acts were preceded by a slow negative-going potential named readiness potential (RP), that begins $500 \mathrm{~ms}$ to about $1,000 \mathrm{~ms}$ before the action. The reported $\mathrm{W}$ was approximately $200 \mathrm{~ms}$ before the response. Thus, the neural preparation for the action began about 300-800 ms before the person consciously made the decision to act. 
The authors concluded that conscious will would thus seem to be a latecomer in the process of choice, rather than the instigator of choice (Libet et al., 1983).

These results contrast our constant feeling of being fully responsible of our actions. Indeed, we feel, at least in a vast number of circumstances, that we have full and conscious access to our intentions and action plans. However, the main results have been confirmed repeatedly (e.g. Haggard and Eimer, 1999; Soon et al., 2008) and there is now experimental evidence suggesting that the sense of volition is not a driving force in the initiation of our behavior. Rather, it seems that the subjective experience of free will is a construction, derived from the brain's motor system producing a movement and somehow 'informing' consciousness of the movement, with the effect that we feel as if the action has been freely chosen (Haggard and Clark, 2003; Hallett, 2007; Kühn and Brass, 2009).

It has been hypothesized recently that the sense of volition is based on retrospective processes (Lau et al., 2007; Banks and Isham, 2009; Kühn and Brass, 2009). For instance, Lau et al. (2007) showed that transcranial magnetic stimulation (TMS) of the pre-supplementary motor area (pre-SMA) $200 \mathrm{~ms}$ after action execution, shifted the perceived time of action closer to the response. Other studies reported that a large part of $\mathrm{W}$ is inferred retrospectively from the response (Banks and Isham, 2009; Kühn and Brass, 2009). In other words, the feeling of consciously intending to act would be influenced by events occurring after the supposed moment of decision. Going back to our decision to turn on the TV, this would mean that some events occurring just after the act of turning on the TV, for instance the button click, would have influenced our feeling of consciously and voluntarily deciding to turn on the TV.

Banks and Isham (2009) recently provided experimental demonstration of this hypothesis, showing that the critical cue for judgment of intention is the perception of the response, thus reversing the assumed causal relation between intention and action. They used a variant of the Libet's task (Libet et al., 1983) in which they gave participants delayed-response feedback to create the illusion that their response was later than it actually was. If the perceived time of action is a prominent factor in judging the beginning of the intention, then a delay in the perceived time of the action would result in a delay in the reported time of $\mathrm{W}$. They found that the reported Ws changed accordingly with the delayed feedback, meaning that $\mathrm{W}$ is based largely on the apparent time of response and not on the motor response or other prior brain events.

In the present study, we wanted to address the issue of what neural mechanisms underlie the inferential process of the conscious intention by recording ERP while subjects are performing the variant of the Libet's task developed by Banks and Isham (2009). If the feeling that we all have, of consciously causing an action, is inferred, at least partially, from something occurring after the action is produced by the subject (Lau et al., 2007; Banks and Isham, 2009; Kühn and Brass, 2009), there must be some neural events accounting for this retrospective timing of conscious intentions. Although previous studies provided empirical demonstrations that the timing of our conscious decisions is influenced by post-action brain processes (Lau et al., 2007), and is based on an inference rather than a perception (Banks and Isham, 2009), little is known about neural mechanisms actually driving this reconstructive process.
Event-related potentials are a useful method to investigate the temporal profile of neural processes underpinning cognitive functions. This method has been utilized previously in the research on free will with the objective of identifying a relationship between the preparatory motor activity (i.e. the RP or the lateralized RP) and the estimated time people become conscious of their own intention to produce a response (Libet et al., 1983; Haggard and Eimer, 1999). For instance, Haggard and Eimer (1999) showed that the reported $\mathrm{W}$ correlates better with the late part of the RP, namely the lateralized RP, than with the RP, as originally reported by Libet et al. (1983). However, we investigated ERPs occurring after participants produced a response using a variant of the Libet's original task (Libet et al., 1983). Delayed auditory feedbacks were provided 5, 20, 40 or $60 \mathrm{~ms}$ after participants' response (i.e. a button press). If the time at which we become conscious of the intention to act, that is the $\mathrm{W}$, is inferred largely by the apparent time of response (i.e. the auditory feedback), then we will find ERP feedback-related components to change across delayed feedbacks.

Our specific hypothesis is that the reconstructive process of conscious decisions is modulated by the activity of an action-monitoring system that involves the medial frontal cortex (Oliveira et al., 2007; Band et al., 2009). Several studies support the idea that the brain has a specific mechanism for monitoring performance (Miltner et al., 1997; Holroyd and Coles, 2002; Yeung and Sanfey, 2004; Oliveira et al., 2007; Band et al., 2009). Converging lines of evidence stress the role of the medial frontal cortex, and in particular the anterior cingulate cortex (ACC), in action monitoring and in the evaluation of behavioral performance (Miltner et al., 1997; Oliveira et al., 2007; Band et al., 2009). It has been shown that the medial frontal cortex is involved in conflict monitoring (Yeung and Sanfey, 2004), error processing (Miltner et al., 1997) and reinforcement learning (Holroyd and Coles, 2002). For instance, the error-related negativity (ERN) and the feedback-related negativity (FRN) are elicited when participants commit an error or received a feedback worse than expected, respectively, and are thought to reflect an increased activity of the ACC.

It has been suggested recently that both FRN and ERN would mirror the activity of a system that detects deviations from expectancy rather than negative feedbacks or performance errors (Oliveira et al., 2007; Band et al., 2009). Consistent with the idea of a general system of action-monitoring, Oliveira et al. (2007) showed that the FRN is elicited when a feedback is different from the expected feedback, regardless of whether it is a positive or a negative one. In a different study, Band et al. (2009) showed that a negative wave peaking in the typical FRN time range was elicited by task-irrelevant action effects, that is, by action-contingent information that was not relevant for the task. These findings support the idea of an actionmonitoring system that not only detects errors and negative feedbacks, but that is sensitive to violations of expectancies. According to this expectancy-deviation hypothesis (Oliveira et al., 2007), the medial frontal cortex would act as a part of a general system that detects deviations from expectancy; the system compares people's expected feedback to the actual feedback and is activated when a mismatch between the two is detected.

The expectancy-deviation hypothesis is in line with a more general theoretical framework of internal models (Wolpert and Ghahramani, 2000; Blakemore et al., 2002). Specifically, the 
'forward model' provides predictions of the sensory consequences of our own actions. Whenever such predictions are confirmed by the afferent sensory information, or in other words, when there is no discrepancy between the model's prediction and the actual sensory consequences of the action, then there is no conflict between sensory predictions and sensory consequences of our actions. On the contrary, if there is a mismatch between the model's prediction and afferent sensory information, then a resolution of the conflict would be required.

A number of studies have shown that people tend to modify their behavioral performance on the basis of false feedbacks, although they are unaware of the mismatch between the sensory predictions and the (false) sensory consequences of the action (Goodale et al., 1994; Blakemore et al., 2002). Under this framework, the shift forward of the reported W found by Banks and Isham (2009) would arise from the increased activity of the action-monitoring system, reflecting the comparison between the model's prediction (i.e. the auditory feedback is expected to be simultaneous to the button press) and the actual sensory consequences of the action (i.e. the auditory feedback is delayed relative to the button press). This, in turn, would result in a 'resolution' of the conflict by adjusting the perceived time of conscious decisions, as measured by the W judgment.

As mentioned above, it has been proposed that feedback- and performance-related negativities reflect the activity of the medial frontal cortex and are elicited when the actual outcome of the performance violates the predicted outcome (Oliveira et al., 1997). Furthermore, an action-effect negativity $\left(N_{\mathrm{AE}}\right)$ is also elicited in case of task-irrelevant feedbacks that are only contingent with the motor action (Band et al., 2009). Here we tested the hypothesis that delayed feedbacks following voluntary movements would elicit larger $N_{\mathrm{AE}}$ as a function of an increase in the temporal mismatch between the actual and the perceived time of response. Our prediction is that an increase in the mismatch between participants' actual response and the delayed feedback would lead to increased activity in the action-monitoring system, resulting in larger $N_{\mathrm{AE}}$ for later delayed feedbacks as compared to earlier delayed feedbacks. In addition, we expected the activity of the action-monitoring system - reflected by the $N_{\mathrm{AE}}$ - to result in a resolution of the conflict by adjusting the timing of conscious decisions to move, thus shifting the reported $\mathrm{W}$ toward the apparent time of response indicated by the delayed auditory feedback. Therefore, if our hypothesis is correct, we should find the amplitude of the $N_{\mathrm{AE}}$ being predictive of the changes in the reported Ws across delayed feedbacks.

Response-feedback not only evoke a negativity over frontocentral areas in the 250 and $350 \mathrm{~ms}$ time range, but also a positive deflection around 300-600 ms after stimulus onset (Overbeek et al., 2005; Waszak and Herwig, 2007; Band et al., 2009). This component, namely P3, is considered to consist of two mutually related components that may be elicited in tandem: the $\mathrm{P} 3 \mathrm{a}$ has a fronto-central distribution, is elicited by deviant or novel stimuli and is considered to reflect the orienting of attention; the P3b has a more posterior distribution and is elicited by infrequently occurring stimuli that are task-relevant, or involve a decision (Waszak and Herwig, 2007). In the current study, feedbacks signaling the button press may elicit a positive waveform. Typically, however, P3 components have been investigated in tasks involving deviant stimuli that differed in terms of physical parameters and/or probability of occurrence (Nittono, 2006; Waszak and Herwig, 2007) and that involved a decision (Knight, 1996; Waszak and Herwig, 2007). On the contrary, in the current study the auditory feedbacks have equal physical parameters, duration, and probability of occurrence and were not related to any decision-making process. Furthermore, it has been proposed that early negative and late positive components reflect two separate action-monitoring systems that differ in terms of the degree of awareness involved (Falkenstein et al., 1991; Kaiser et al., 1997; Nieuwenhuis et al., 2001; Overbeek et al., 2005). This idea is supported by empirical observations that the degree of awareness of an error covaries with the amplitude of the P3, but not with the amplitude of the preceding negative component (Kaiser et al., 1997; Nieuwenhuis et al., 2001). Consistent with these observations, Nieuwenhuis et al. (2001) suggested that the two components reflect the activity of two distinct action-monitoring processes, of which only that reflected by the $\mathrm{P} 3$ is associated with conscious error recognition. In the current study, we expected participants to be unaware of the temporal mismatch between the response and the delayed feedback. Therefore, we expected only the $N_{\mathrm{AE}}$ to be modulated by the experimental manipulation, whereas the $\mathrm{P} 3$ was expected to be insensitive to the experimental manipulation.

\section{MATERIALS AND METHODS PARTICIPANTS}

Sixteen undergraduates ( 7 females, 9 males; age range 19-24 years) from the University of Padova volunteered for the present study. All participants had normal or corrected-to-normal vision, gave informed written consent and were debriefed at the end of the experiment. Participants were paid $8 €$ for taking part in the experiment.

\section{APPARATUS AND PROCEDURE}

The experiment followed the procedure used by Banks and Isham (2009) and is based on the method used by Libet et al. (1983). Participants sat in front of a computer screen. In each trial, a cursor on the computer screen moved in a clockwise direction around a clock face, completing three revolutions in $8.1 \mathrm{~s}$. The clock was $90 \mathrm{~mm}$ in diameter with 60 evenly spaced spots. Presentation of the clock and recording of responses was controlled by E-Prime 1.1 software.

Participants were requested to fixate the center of the clock and to rest their right index finger on the response button, which was the keyboard spacebar. The participant's hand was not visible to the participant. Participants were instructed to press the button spontaneously and suddenly at a time of their own choosing, following at least one rotation of the cursor. They were asked not to plan the time of the button press and were told that they could choose not to make a button press in any trial. Participants were explicitly told that an auditory feedback was delivered simultaneously with each button press. In fact, the computer emitted a $200-\mathrm{ms}$ beep by a computer-generated random sequence at 5, 20, 40, or $60 \mathrm{~ms}$ right after the button press. After the button press the cursor rotated for a random interval between 800 and 1,500 ms and then stopped. Then, participants were asked to report the position of the cursor at the instant they made the decision to respond. 
Participants performed a practice session of 16 trials. Then, the experimental session started. There were 40 trials at each delay, for a total of 160 trials, administered in two separate blocks. Some trials did not yield data because participants chose not to respond $(<1 \%)$. At the end of the experimental session, we asked participants whether they experienced a temporal mismatch between button press and the auditory feedback. None of the participants acknowledged a temporal mismatch between the two events.

\section{EEG RECORDING}

Scalp voltages were recorded using a 59-channel electrocap with Ag/AgCL electrodes, arranged according to the 10-20 system. A frontal electrode (AFz) was connected to the ground. Mastoids served for reference and electrode impedance was kept under $10 \mathrm{~K} \Omega$ for all recordings. Vertical and horizontal ocular movements were also recorded. Signals were amplified and digitized with a Neuroscan system at a sampling rate of $500 \mathrm{~Hz}$. Off-line analyses were performed with Brain Vision Analyzer. The signal was off-line filtered (high pass 0.01 and low pass $30 \mathrm{~Hz}, 24 \mathrm{~dB}$ / octave attenuation). Ocular movements' artefacts were corrected using the algorithm provided by Brain Vision Analyzer (Gratton et al., 1983). Electrical recordings were segmented in epochs starting $100 \mathrm{~ms}$ before the presentation of the auditory feedback and lasting until $900 \mathrm{~ms}$ after its onset. The epochs were aligned to the $100 \mathrm{~ms}$ pre-stimulus baseline relative to the auditory feedback. Epochs affected by artefacts $( \pm 80 \mu \mathrm{V})$ were excluded from averaging (rejected epochs $\sim 5 \%$ ).

\section{EMG RECORDING}

The electromyographic signal (EMG) was recorded from the flexor carpi radialis and nearby muscles by two electrodes pasted on the velar surface of the right forearm. The onset of the EMG was determined by an algorithm provided by Van Boxtel et al. (1993) followed by a visual re-check of the EMG onset search procedure. EMG onset was measured as the first point at which the EMG signal reached 3 standard deviations from the baseline.

\section{RESULTS \\ BEHAVIORAL PERFORMANCE}

Trials with no responses provided by the participant, and with reported $\mathrm{W}$ exceeding \pm 3 standard deviations from individual averages, were considered as errors and were discarded from the data analysis $(\sim 2 \%)$. One participant showed a high rate of trials exceeding this criterion $(>1 / 3)$ and was therefore excluded from further analyses. A repeated-measure ANOVA was performed with delay $(5,20,40$, and $60 \mathrm{~ms})$ as within subjects' factor and the reported $\mathrm{W}$ as dependent variable. We found a significant effect of the delay factor $\left(F(3,42)=8.26, p=0.004, \eta_{p}{ }^{2}=0.37\right)$. The averaged reported Ws at delays of 5, 20, 40, and $60 \mathrm{~ms}$ were $-127,-111$, -102 , and $-101 \mathrm{~ms}$, respectively, relative to time of response (see Figure 1). The observed power was 0.88 .

\section{EVENT-RELATED POTENTIALS}

For the analyses, only trials in which participants provided a response were used. Figure 2A shows grand average ERPs following delayed auditory feedbacks in three different electrodes. We quantified $N_{\mathrm{AE}}$ amplitude in the averaged waveforms for each participant

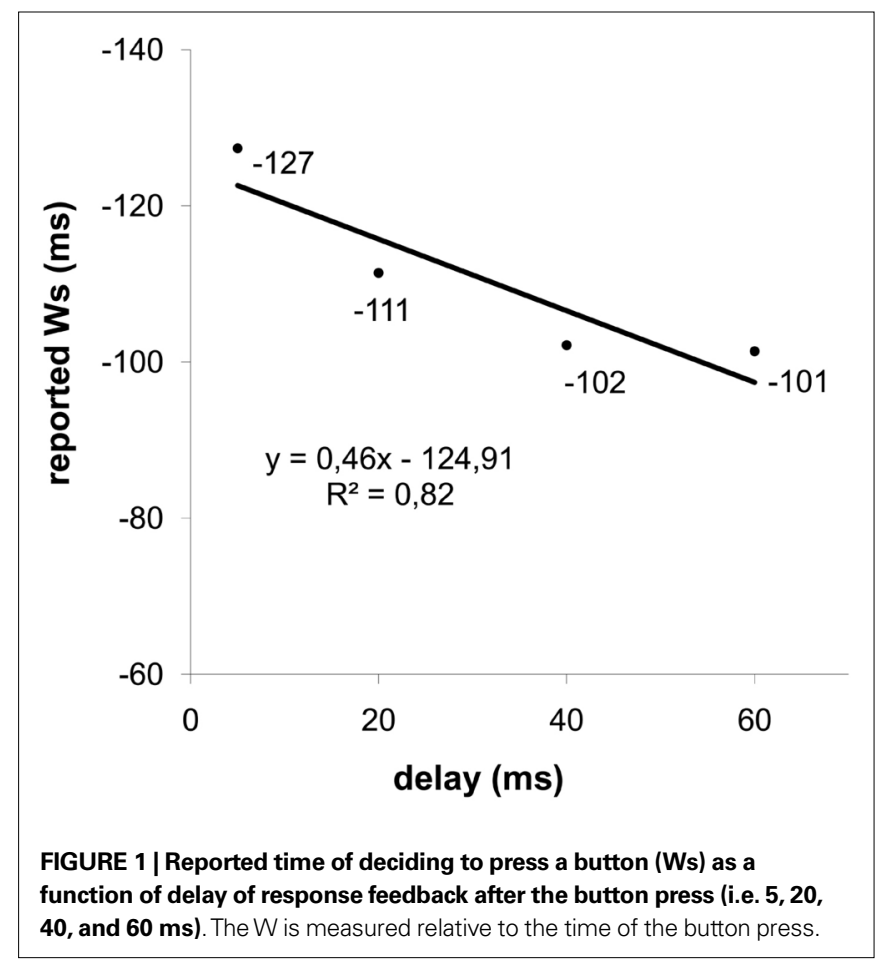

as the base-to-peak difference between the average voltage within 260-300 ms after stimulus onset and the average voltage of the immediately preceding positive peak in the 180-220 ms time window. We used the average amplitude of the $N_{\mathrm{AE}}$ instead of peak amplitude because there were no clear maxima in the selected time range. We also measured $\mathrm{P} 3$ amplitude. The amplitude of the $\mathrm{P} 3$ was quantified as the most positive peak in the waveform in the 300-400 ms period after stimulus onset, as compared to the $100 \mathrm{~ms}$ pre-stimulus baseline.

Component amplitudes were preliminarily entered into a repeated-measure ANOVA with electrode site $(\mathrm{Fz}, \mathrm{FCz}, \mathrm{Cz}, \mathrm{CPz}, \mathrm{Pz})$ as within subjects' factor in order to identify scalp locations in which $N_{\mathrm{AE}}$ and P3 showed their maximum amplitude. The GreenhouseGeisser correction for violations of the assumption of sphericity was applied where appropriate. The analyses revealed a significant effect of electrode site, both for the $N_{\mathrm{AE}}(F(4,56)=13.61, p<0.0001$, $\left.\eta_{\mathrm{p}}{ }^{2}=0.49\right)$ and for the P3 $\left(F(4,56)=5.19, p=0.001, \eta_{\mathrm{p}}{ }^{2}=0.27\right)$. $N_{\mathrm{AE}}$ showed a central scalp distribution, with a maximum over $\mathrm{Cz}$, followed by $\mathrm{FCz}, \mathrm{CPz}, \mathrm{Fz}$, and $\mathrm{Pz}$. P3 was more pronounced over fronto-central electrodes, with a maximum over $\mathrm{FCz}$, followed by $\mathrm{Fz}, \mathrm{Cz}, \mathrm{CPz}$, and $\mathrm{Pz}$.

Visual inspection of grand-averaged ERPs revealed a positive wave over fronto-central sites, namely the P200, occurring within 150-250 ms time window after stimulus onset. In order to exclude that this early component was sensitive to the experimental manipulation, we determined P200 amplitude through a base-to-peak procedure as the positive peak within 150-250 time window relative to the preceding negative peak in the 50-150 time window. A repeated-measure ANOVA was performed with feedback delay (5, 20, 40, $60 \mathrm{~ms}$ ) and electrode site $(\mathrm{Fz}, \mathrm{FCz}, \mathrm{Cz}, \mathrm{CPz}, \mathrm{Pz})$ as within subjects' factors and the amplitude of the P200 as the dependent variable. The analysis showed a significant effect of electrode site 


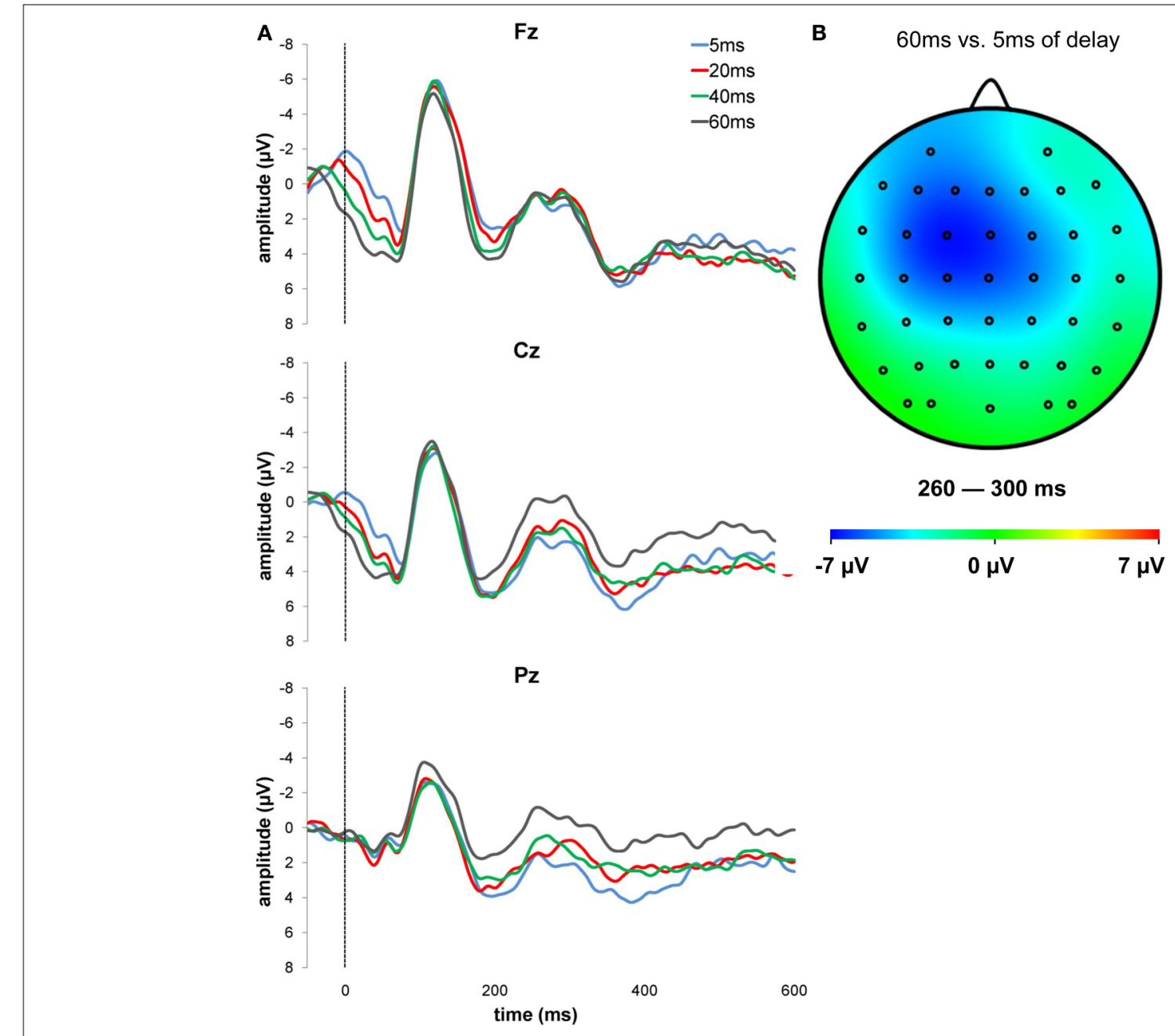

FIGURE 2 | (A) Grand-averaged ERPs for the different delays of the auditory feedback at Fz, Cz, and Pz. The waveforms are time-locked to the delayed feedback onset, that is, represented in the figure by the vertical line. (B) Topographic difference in the $\mathrm{N}_{\mathrm{AE}}$ time range (260-300 ms) for 60-5 ms of feedback delay is displayed.

$\left(F(4,56)=7.81, p<0.0001, \eta_{\mathrm{p}}{ }^{2}=0.36\right)$, with $\mathrm{P} 200$ being larger over $\mathrm{FCz}$, followed by $\mathrm{Fz}, \mathrm{Cz}, \mathrm{CPz}$, and $\mathrm{Pz}$, but no effect of feedback delay $(p=0.77)$ and no interaction $(p=0.22)$.

To test our prediction that the $N_{\mathrm{AE}}$ would increase as a function of feedback delay, the $N_{\mathrm{AE}}$ amplitude over $\mathrm{Cz}$ was subjected to a repeated-measure ANOVA with the feedback delay $(5,20,40$, $60 \mathrm{~ms}$ ) as within subjects factor. The analysis yielded a significant effect $\left(F(3,42)=3.42, p=0.026, \eta_{\mathrm{p}}{ }^{2}=0.20\right)$, with larger $N_{\mathrm{AE}}$ amplitude for more delayed feedbacks $-2.87,-4.15,-4.22$, and $-5.06 \mu \mathrm{V}$ for feedbacks delayed by 5, 20, 40 and $60 \mathrm{~ms}$, respectively; see Figure 3). Figure 2B shows the topographical distribution map of the difference in the signal between the condition with the larger (i.e. $60 \mathrm{~ms}$ ) and the smaller $N_{\mathrm{AE}}$ (i.e. $5 \mathrm{~ms}$ ). This result confirms our prediction that the $N_{\mathrm{AE}}$ is sensitive to the temporal mismatch between the actual response and the delayed auditory feedback. More delayed auditory feedbacks are associated with larger $N_{\mathrm{AE}}$ amplitudes, suggesting that the amplitude of the $N_{\mathrm{AE}}$ increases as a function of the delay of the feedback. The linear component of the feedback delay effect was significant $(F(1,14)=6.16, p=0.026$, $\left.\eta_{\mathrm{p}}^{2}=0.31\right)$.
P3 amplitude over FCz was subjected to a repeated-measure ANOVA with the feedback delay $(5,20,40,60 \mathrm{~ms})$ as within subjects' factor. The analysis yielded a marginal effect $(F(3,42)=2.65$, $p=0.061$ ), with smaller P3 amplitudes for more delayed feedbacks $(6.36,6.58,5.65$, and $4.63 \mu \mathrm{V}$ for feedbacks delayed by $5,20,40$ and $60 \mathrm{~ms}$, respectively).

\section{$\boldsymbol{N}_{A E}$ and reported $\boldsymbol{W}$}

Next, we performed a hierarchical multiple linear regression analysis to test the prediction that the shift of the reported $\mathrm{W}$ toward the apparent time of response is associated with the increased amplitude of the NAE induced by the delay of the auditory feedback. The amplitude of $N_{\mathrm{AE}}$ for each participant and for each delayed feedback was entered at the first block and the level of feedback delay $(5,20,40$ and $60 \mathrm{~ms})$ was entered at the second block; reported Ws were the dependent variable. We found that the amplitude of the $N_{\mathrm{AE}}$ predicted the reported $\mathrm{W}$ significantly $\left(\beta=-0.32, t=-2.58, p=0.012 ; F(1,58)=6.66, R^{2}=0.10\right)$. When the proportion of variance in the reported $\mathrm{W}$ explained by $N_{\mathrm{AE}}$ amplitude was removed from the model, the feedback delay factor 


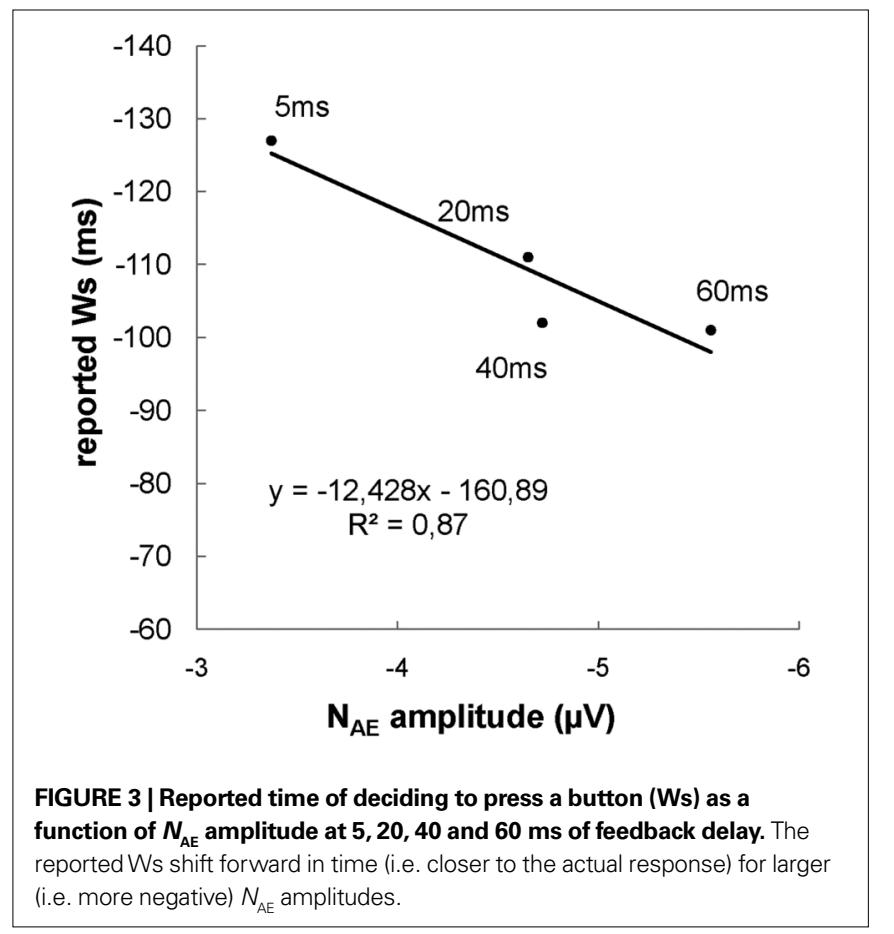

failed to predict the change in the reported $\mathrm{W}(p=0.44)$. This result strongly indicates that changes in the reported $\mathrm{W}$ are indeed associated with changes in the NAE amplitude, regardless of the delay of the feedback.

\section{EMG DATA}

The EMG onset was measured as the point of the first steep increase in the EMG record in the $400 \mathrm{~ms}$ interval preceding the button press. The EMG preceded the button press by $\sim 99 \mathrm{~ms}$, with little variability between delays $(99,100,100$, and $98 \mathrm{~ms}$ for the four delays, respectively). In order to assess whether the onset of the movement was related to the $\mathrm{W}$, a linear regression analysis was performed with the EMG onset as predictor and the reported Ws as dependent variable. The analysis showed that the relationship is not significant $(p=0.42)$, suggesting that the $\mathrm{W}$ is not related to the actual onset of the movement.

\section{DISCUSSION}

Recent findings showed that the subjective experience of conscious decisions is influenced by events occurring after a motor response is actually produced (Lau et al., 2007; Banks and Isham, 2009; Kühn and Brass, 2009). The feeling of causing an action consciously and deliberately seems to be, at least in part, based on a reconstructive process that depends largely on post-action events. Under this view, external cues related to the consequence of our actions, and compatible with our sensory prediction of the consequences of the action (Wolpert et al., 1995), are processed by the brain and influence the feeling that we intended that action consciously and voluntarily. In these terms, it has been argued that the feeling that we have free will is, at least partially, dependent on an a posteriori reconstruction (Lau et al., 2007; Banks and Isham, 2009; Kühn and Brass, 2009).
The aim of the present study was to investigate psychophysiological mechanisms underlying the reconstructive process of conscious decisions. In order to do so, we recorded ERPs while participants were performing a variant of the classical Libet's task (Banks and Isham, 2009). So far, ERP research on free will have focused on preparatory activity (i.e. RP or lateralized RP) occurring before an action was produced (Libet et al., 1983; Haggard and Eimer, 1999). On the contrary, in the present study we focused on post-action brain events that may be related to the reported time of when a conscious decision to execute an action was made. In order to do so we asked participants to press a button at will and at the same time to monitor a red spot moving around a clock face. They were then asked to report the location of the spot when they had the first intention to move. This measure is called the $\mathrm{W}$ judgment (Libet et al., 1983). Participants were also told that an auditory feedback, simultaneous to the button press, signaled that the response was provided. Indeed, unbeknown to the participants, the auditory feedback was not temporally overlapping the actual response, but could be delayed forward by $5,20,40$, and $60 \mathrm{~ms}$.

It has been suggested that people have an internal representation of the sensory consequences of their own actions (Wolpert and Ghahramani, 2000; Blakemore et al., 2002) and that they use these internal representations to form predictions about the outcome of behavior. Wolpert and Ghahramani (2000) suggest that two kinds of internal models lie behind these functions. The 'forward model' uses efference copy to predict the sensory consequences of motor commands whenever a movement is made. By contrast, the 'inverse model' provides motor commands that are necessary to achieve a desired outcome. An important aspect of the forward model is that it predicts the sensory consequences of movement and compares this with the actual feedback (Blakemore et al., 2002). This comparison occurs after a movement is made, and people are normally unaware of the actual state of the motor system and the actual sensory consequences of a movement. Furthermore, we are also unaware of the results of the comparison between the predicted and actual sensory feedback, as long as the desired state is achieved successfully. If the predictions made by the forward model are confirmed by the afferent sensory information, then there is no discrepancy between the model's prediction and the actual sensory consequences and, therefore, there is no conflict between the sensory predictions and the sensory consequences of the actions. On the contrary, a mismatch between the model's prediction and afferent sensory information would require a resolution of the conflict (Goodale et al., 1994; Blakemore et al., 2002).

In line with the idea that people form internal representations of the consequences of the actions, the expectancy-deviation hypothesis (Oliveira et al., 2007; Band et al., 2009) poses that feedback- and performance-related ERP components, such as the FRN and the ERN, reflect the activity of the medial frontal cortex and are elicited when the actual outcome of the performance violates the predicted outcome (Oliveira et al., 2007; Band et al., 2009). In the present study, participants were told explicitly that the auditory feedback was delivered simultaneously with the button press. Therefore, we assumed that participants expected the sensory consequences of the action to be simultaneous with the button press. With this in mind, the auditory delayed feedbacks signaling the motor response later than it actually was, may be considered as a form of mismatch 
between the predictions of the sensory consequences and the actual sensory consequences of the action. Therefore, our first hypothesis was that the delay of the response feedback would result in an increased activity of the action-monitoring system (Oliveira et al., 2007; Band et al., 2009), reflecting the comparison between the model's prediction and the actual sensory consequences of the action.

At a behavioral level, we replicated the finding that the delay of the feedbacks did influence the reported W (see Figure 1). Participants shifted the reported time at which they had the intention to press the button (i.e. the $\mathrm{W}$ ) forward in time, according with the delayed feedbacks. These results are in line with those obtained by Banks and Isham (2009) and are in accordance with the reconstructive hypothesis of the sense of free will (Aarts et al., 2005; Lau et al., 2007; Banks and Isham, 2009; Kühn and Brass, 2009). However, the feedback delay was not completely reflected in the reported $\mathrm{W}$. If the report of $\mathrm{W}$ was locked perfectly to the feedback, the slope relating $\mathrm{W}$ to delay of feedback would have been equal to 1 . The slope of 0.46 suggests that constant cues unrelated to the tone affected the perception of response time. Indeed, it is not possible to exclude that somatosensory and tactile cues, as well as the effort at the beginning of the button press, may in fact have had an impact on the perceived time of response.

We found no relationship between the $\mathrm{W}$ and the actual onset of the movement as recorded by the EMG. This suggests that changes in the subjective timing of conscious decisions were not attributable to changes in the actual onset of the movement, as measured by the EMG activity.

As regards ERP components, we found that the auditory feedbacks following voluntary button presses elicited a negative wave in the 260-300 ms time range. We referred to this component as action-effect negativity $\left(N_{\mathrm{AE}}\right)$, in accordance with a previous study by Band et al. (2009). Crucially, our experimental manipulation had a significant effect on the $N_{\mathrm{AE}}$ Its amplitude was maximal over fronto-central scalp locations and was enhanced as a function of the delay of the feedback signaling the time of response (see Figure 2). In other words, the longer the delay between the actual response and the auditory feedback, the greater the $N_{\mathrm{AE}}$ amplitude. This result supports our hypothesis that the activity of the action-monitoring system, reflected in the current study by the $N_{\mathrm{AE}}$, is enhanced when delayed feedbacks signal that the response was later than it actually was. Interestingly, although the $N_{\mathrm{AE}}$ was maximal over $\mathrm{Cz}$, the topography of the difference between 60 and $5 \mathrm{~ms}$ of feedback delay (see Figure 2B) showed a lateralization over the controlateral fronto-central sites. We can speculate that this lateralization is due to the motor lateralization induced by response execution processes. It has been suggested that the propagation of the primary motor cortex activity towards premotor areas (Roger et al., 2010) may result in a lateralization of performance-related negativities following motor responses.

The relationship between the delay of the feedback and $N_{\mathrm{AE}}$ amplitude may not be linear, as indicated by Figures 2 and 3 showing that $N_{\mathrm{AE}}$ amplitudes with feedbacks delayed by 20 or $40 \mathrm{~ms}$ are almost overlapping. This suggests that other feedbacks from the environment, such as somatosensory and tactile cues, as well as the effort at the beginning of the button press, may be processed by the action-monitoring system and have an impact on the amplitude of the $N_{\mathrm{AE}}$. In other words, the $N_{\mathrm{AE}}$, as well as the reported $\mathrm{W}$, may reflect the integration of several action-related feedbacks - not just the effect of the delayed auditory feedback.

The auditory feedback also evoked a positive component, namely the P3, peaking at around $360 \mathrm{~ms}$ after stimulus onset. A number of studies showed that P3 amplitude is involved in the performancemonitoring process (see Overbeek et al., 2005 for a review); this component is typically larger for stimuli that deviate from expectations, such as errors or unexpected feedbacks. The lack of effect on the P300 amplitude strengthens the point the participants were unaware of the feedback delay, given that this component is sensitive to stimulus awareness (Overbeek et al., 2005). Unexpectedly, we found P3 amplitude being smaller for more delayed feedbacks - and thus more deviating from the predictions of the sensory consequences - as compared with less delayed feedbacks. However, the effect was not significant; it seems likely that the variability in the preceding $N_{\mathrm{AE}}$ contributed to the marginal effect found for the P3 amplitude.

A second hypothesis was that the activity of the actionmonitoring system, reflected by the $N_{\mathrm{AE}}$, is involved in the reconstructive process of conscious decisions. This prediction was based on previous studies showing that when there is a conflict between the predictions of the sensory consequences and the actual sensory consequences of an executed voluntary action, individuals tend to adjust their behavioral performance in order to reduce the conflict (Goodale et al., 1994; Blakemore et al., 1999). In the current study, we used a Libet task (Libet et al., 1983; Banks and Isham, 2009) and asked participants to report the time (i.e. W) of the conscious decision of a voluntary button press. If the hypothesis that the actionmonitoring system is involved in the timing of conscious decisions is correct, we would expect the reported W to be linked to the increased activity of the action-monitoring system, here reflected by the $N_{\mathrm{AE}}$. We found that the amplitude of the $N_{\mathrm{AE}}$ predicted the reported W significantly, with increased $N_{\mathrm{AE}}$ amplitudes resulting in reported Ws closer to the button press. Crucially, when controlled for $N_{\mathrm{AE}}$ amplitude, the feedback delay factor failed to predict the reported $\mathrm{W}$, indicating that changes in the reported $\mathrm{W}$ are indeed associated with changes in the amplitude of the $N_{\mathrm{AE}}$, and not just to the delay of the auditory feedback. This result is consistent with our hypothesis that the activity of the action-monitoring system, reflected in the current study by the amplitude of the $N_{\mathrm{AE}}$, plays a role in the reconstructive process of our conscious decisions, as measured by the $\mathrm{W}$ judgment.

A remaining question is how the $N_{\mathrm{AE}}$ obtained in the current study should be classified with regard to the fronto-central negativities peaking around $250 \mathrm{~ms}$ (see Folstein and Van Petten, 2008 for a review). For instance, the mismatch negativity (MMN; Näätänen and Alho, 1997) is a negative-going component that follows deviant auditory stimuli. However, it is only elicited when there is a substantial difference between the overall probability of a standard and a deviant auditory stimulus, whereas in the current study the delayed auditory feedbacks have an equal probability of 0.25 . Another N2 component, namely the control-related N2, is elicited by stimuli inducing response conflict and/or requiring response inhibition (Folstein and Van Petten, 2008). In the current study, it is unlikely that the delayed auditory feedbacks induced response conflict or response inhibition, since the response was already executed before the presentation of the 
auditory stimulus. Regardless of the exact classification of the $N_{\mathrm{AE}}$, however, this study supports the idea of a system that detects deviation from expectancies (Oliveira et al., 2007; Band et al., 2009).

To summarize we showed that reported time of decisions was predictable on the basis of the amplitude of a negative component related to the action-effects (Band et al., 2009), occurring 260-300 ms after the presentation of an auditory feedback. These results suggest that when people are asked to report when they made the decision to execute an action, they are strongly influenced by the consequences of the action. Furthermore, we showed that this retrospective process relies on the activity of an action-monitoring system that, it has been argued, involves the medial frontal cortex (Miltner et al., 1997; Holroyd and Coles, 2002; Oliveira et al., 2007; Band et al., 2009).

\section{CURRENT FINDINGS AND THE LIBET PARADIGM}

There is an extensive debate on the question as to what extent results from this kind of experiment have implications for our understanding of volition and intent (Hallett, 2007; Pocket and Miller, 2007). Our results indicate that if asked to report when they decided to execute an action, people rely - presumably at an implicit level - on the perceived sensory consequences of the action. With respect to the free will debate, these findings support the idea that the Libet paradigm may not be the best approach to investigate conscious intentions of motor actions. It is not possible to exclude that people use systematic strategies in this task, either consciously or in an automatic manner, to judge the time of conscious decisions. For instance, they might remember the position of the clock at the time of the feedback (e.g. the auditory tone and/or somatosensory and tactile feedbacks), and then infer that the decision 'must' have taken place somewhere before. In any case, if people infer, at least partially, the time they decided to act from the consequences of the action (e.g. a tone signaling the response), then the reported $\mathrm{W}$ used in the Libet paradigm may not be a reliable measure for the investigation of when a decision to act enters subjective awareness (for a review on limitations of the Libet paradigm refer to Pockett and Miller, 2007).

\section{CONCLUSION}

In the present work, a small disruption of the temporal parameter of an auditory feedback, caused people to shift forward in time the $\mathrm{W}$ judgment, suggesting that the timing of our conscious

\section{REFERENCES}

Aarts, H., Custers, R., and Wegner, D. M. (2005). On the inference of personal authorship: enhancing experienced agency by priming effect information. Conscious. Cogn. 14, 439-458.

Banks, W. P., and Isham, E. A. (2009). We infer rather than perceive the moment we decided to act. Psychol. Sci. 20, 17-21.

Band, G. P. H., van Steenbergen, H., Ridderinkhof, K. R., Falkenstein, M., and Hommel, B. (2009). Action-effect negativity: irrelevant action effects are monitored like relevant feedback. Biol. Psychol. 82, 211-218.

Baumeister, R. F. (2008). Free will in scientific psychology. Perspect. Psychol. Sci. 3, 14-19.
Blakemore, S. J., Frith, C. D., and Wolpert, D. M. (1999). Spatiotemporal prediction modulates the perception of selfproduced stimuli. J. Cogn. Neurosci. 11, 551-559.

Blakemore, S. J., Wolpert, D. M., and Frith, C. D. (2002). Abnormalities in the awareness of action. Trends Cogn. Sci. 6, 237-242.

Falkenstein, M., Hohnsbein, J., Hoormann, J., and Blanke, L. (1991). Effects of crossmodal divided attention on late ERP components. II. Error processing in choice reaction tasks. Electroencephalogr. Clin. Neurophysiol. 78, 447-455.

Folstein, J. R., and Van Petten, C. (2008). Influence of cognitive control and mismatch on the $\mathrm{N} 2$ component of

decisions is at least partially based on a reconstructive process (Banks and Isham, 2009; Kühn and Brass, 2009). Our participants were unaware of the delay, and this provides further evidence that the comparison between the intended sensory consequences and the actual sensory consequences is unavailable to awareness (Wolpert and Ghahramani, 2000; Blakemore et al., 2002). Under this view, the shift in the $\mathrm{W}$ judgment may be the result of a sort of expectancy violation related to the predicted sensory feedback of our action. The fact that participants were unaware of the delay suggests that the action-monitoring system operates at an implicit level and this is in line with previous data showing that people are unaware of the mismatch, even with longer delays between the predicted and the actual sensory consequence of the action (Blakemore et al., 1999). Nevertheless, it is possible that such an implicit monitoring system has a kind of temporal threshold after which feedback is not considered as belonging to the action anymore. However, this idea is very speculative and would need further investigation.

Our interpretation of the result, is that the temporal mismatch between the predicted and the actual sensory feedback was detected by the action-monitoring system (Oliveira et al., 2007; Band et al., 2009), as reflected by the $N_{\mathrm{AE}}$ amplitude, and caused a shift in the subjective timing of when the action was decided. To our knowledge, this is the first study employing ERPs recording for investigating psychophysiological mechanisms underlying reconstructive processes of conscious decision. Changes in the reported $\mathrm{W}$ were related to what we called $N_{\mathrm{AE}}$, a negative potential involved in response and feedback- and action-monitoring (Oliveira et al., 2007; Folstein and Van Petten, 2008; Band et al., 2009). Taken together these findings indicate that the reported time of conscious decisions are influenced strongly by the consequences of our actions, that are constantly monitored by a dedicated system involving the medial frontal cortex.

\section{ACKNOWLEDGMENTS}

This work was supported by the Centro Universitario Internazionale, Monte San Savino, Italy, and the Cassa di Risparmio di Padova e Rovigo (Cariparo), Italy. We thank Sandro Bettella for technical support, and Ramona Guarino and Alessio Lombardi for data collection and analysis.

the ERP: a review. Psychophysiology 45, 152-170.

Goodale, M. A., Jacobson, L. S., Milner, A. D., Perret, D. I., Benson, P. J., and Hietanen, J. K. (1994). The nature and limits of orientation and pattern processing supporting visuomotor and control in a visual form agnosic. J. Cognitive Neurosci. 6, 46-56.

Gratton, G., Coles, M. G.H., and Donchin, E. (1983). A new method for off-line removal of ocular artifact. Electroen. Clin. Neuro. 55, 468-484.

Haggard, P., and Clark, S. (2003). Intentional action: conscious experience and neural prediction. Conscious. Cogn. 12, 695-707.

Haggard, P., and Eimer, M. (1999). On the relation between brain potentials and the awareness of voluntary movements. Exp. Brain Res. 126, 128-133.

Hallett, M. (2007). Volitional control of movement: the physiology of free will. Clin. Neurophysiol. 118, 1179-1192.

Haynes, J., Sakai, K., Rees, G., Gilbert, S., Frith, C., and Passingham, R. (2007). Reading hidden intentions in the human brain. Curr. Biol. 17, 323-328.

Holroyd, C. B., and Coles, M. G. H. (2002). The neural basis of human error processing: reinforcement learning, dopamine, and the errorrelated negativity. Psychol. Rev. 109, 679-709.

Kaiser, J., Barker, R., Haenschel, C., Baldeweg, T., and Gruzelier, J. H. (1997). Hypnosis and event-related potential correlates of error processing 
in a Stroop-type paradigm: a test of the frontal hypothesis. Int. J. Psychophysiol. 27, 215-222.

Knight, R. (1996). Contribution of human hippocampal region to novelty detection. Nature 383, 256-259.

Kühn, S., and Brass, M. (2009). Retrospective construction of the judgment of free choice. Conscious. Cogn. 18, 12-21.

Lau, H. C., Rogers, R. D., and Passingham, R.E. (2007). Manipulating the experienced onset of intention after action execution. J. Cognitive Neurosci. 19, 81-90.

Libet, B., Gleason, C.A., Wright, E. W., and Pearl, D. K. (1983). Time of conscious intention to act in relation to onset of cerebral activity (readiness-potential). The unconscious initiation of a freely voluntary act. Brain 106, 623-642.

Miltner, W. H. R., Braun, C. H., and Coles M. G. H. (1997). Event-related brain potentials following incorrect feedback in a time-estimation task: evidence for a "generic" neural system for error detection. J. Cogn. Neurosci. 9, 788-798.

Näätänen, R., and Alho, K. (1997). Mismatch negativity $(\mathrm{MMN})-$ The measure for central sound representation accuracy. Audiol. Neurootol. 2, 341-353.

Nieuwenhuis, S., Ridderinkhof, K. R., Blom, J., Band, G. P. H., and Kok, A. (2001). Error-related brain potentials are differentially related to awareness of response errors: evidence from an antisaccade task. Psychophysiology 38, 752-760.

Nittono, H. (2006). Voluntary stimulus production enhances deviance processing in the brain. Int. J. Psychophysiol. 59, 15-21.

Oliveira, F. T. P., McDonald, J. J., and Goodman, D. (2007). Performance monitoring in the anterior cingulate is not all error related: expectancy deviation and the representation of action-outcome associations. J. Cogn. Neurosci. 19, 1994-2004.

Overbeek, T. J. M., Nieuwenhuis, S., and Ridderinkhof,K.R.(2005).Dissociable components of error processing. J. Psychophsiol. 19, 319-329.

Pockett, S., and Miller, A. (2007). The rotating spot method of timing subjective events. Conscious. Cogn. 16, 241-254.
Roger, C., Bénar, C. J., Vidal, F., Hasbroucq, T., and Burle, B. (2010). Rostral cingulate zone and correct response monitoring: ICA and source localization evidences for the unicity of correctand error-negativities. NeuroImage doi:10.1016/j.neuroimage.2010.02.005

Soon, C. S., Brass, M., Heinze, H.-J., and Haynes, J.-D. (2008). Unconscious determinants of free decisions in the human brain. Nat. Neurosci. 11, 543-545.

Van Boxtel, G. J. M., Geraats, L. H. D., Van den Berg-Lenssen, M. M. C., and Brunia, C. H. M. (1993). Detection of EMG onset in ERP research. Psychophysiology 30, 405-412.

Waszak, F., and Herwig, A. (2007). Effect anticipation modulates deviance processing in the brain. Brain Res. 1183, 74-82.

Wolpert, D. M., and Ghahramani, Z. (2000). Computational principles of movement neuroscience. Nat. Neurosci. 3, 1212-1217.

Wolpert, D. M., Ghahramani, Z., and Jordan, M. I. (1995). An internal model for sensorimotor integration. Science 269, 1880-1882.
Yeung, N., and Sanfey, A. G. (2004). Independent coding of reward magnitude and valence in the human brain J. Neurosci. 24, 6258-6264.

Conflict of Interest Statement: The authors declare that the research was conducted in the absence of any commercial or financial relationships that could be construed as a potential conflict of interest.

Received: 09 December 2009; paper pending published: 17 February 2010; accepted: 19 April 2010; published online: 14 May 2010.

Citation: Rigoni D, Brass $M$ and Sartori $G$ (2010) Post-action determinants of the reported time of conscious intentions. Front. Hum. Neurosci. 4:38. doi: 10.3389/fnhum.2010.00038

Copyright (C) 2010 Rigoni, Brass and Sartori. This is an open-access article subject to an exclusive license agreement between the authors and the Frontiers Research Foundation, which permits unrestricted use, distribution, and reproduction in any medium, provided the original authors and source are credited. 